Key words: Schizophrenia, Rehabilitation, Well-

being, Caregiver, Family.

\title{
The effect of rehabilitation of schizophrenic patients on their family atmosphere and the emotional well-being of caregivers
}

\author{
Evi Koukia, RN MSc* \\ Michael G. Madianos, MD MPH** \\ * Dr Nursing. Department of Psychiatry \\ Eginition Hospital Medical School University \\ of Athens \\ ** DrMed. Professor of Psychiatry Head of \\ Community Mental Health Center \\ Department of Mental Health/Behavioural \\ Sciences, School Health Sciences, Fac. of \\ Nursing. University of Athens \\ GREECE
}

\footnotetext{
ABSTRACT - Background: In family members with a schizophrenic patient emotional problems, stressful situations, significant life changes are often observed. On the other hand rehabilitation of these patients is an integral part of their aftercare and community tenure.

Aims: In this study the effect of the participation of schizophrenic patients in rehabilitation programmes, on the atmosphere of their families and the emotional well-being of their caregivers are examined.

Method: Sixty six caregivers of patients in rehabilitation (group A) were compared with seventy caregivers of patients, not attending any rehabilitation programme (group B), in terms of their family atmosphere and emotional well-being, assessed by the Family Atmosphere Scale and the CES-D scale respectively.

Results: The family atmosphere of the patients who were participating in a rehabilitation programme was found to be more positive (higher degree of patient's acceptance, autonomy and compliance as well as fewer economic problems) than that of their counterparts, not in rehabilitation. Group A caregivers were also found to exhibit less depressive symptomatology.

Conclusions: It seems that psychosocial rehabilitation and its vanous therapeutic activities has a positive impact on several components of family atmosphere of the participating patients and the emotional status of their caregivers.
} 


\section{Introduction}

Various studies undertaken by mental health clinicians and social scientists have focused on specific characteristics of the families of schizophrenic patients ie roles, communication patterns, transactions, stressful events and burden placed on the health caregivers of the family. Based upon these studies, the importance of family atmosphere in the course of schizophrenia was established (Brown et al. 1972, Vaughn \& Leff 1976, Platt 1985, Madianos et al. 1987, Fadden et al. 1987, Schene et al. 1998, Laidlaw et al. 2002).

Additionally, since the 1960s, when the first community based psychiatric aftercare programmes were introduced, several investigators examined the effects of those programmes on the patient's psychosocial functioning and community tenure, as well as on specific familial factors (Hogarty et al. 1973, Madianos \& Economou 1988, Tomaras et al. 1988, Dyck et al. 1999).

The majority of these studies have shown that overall functioning of psychotic patients affects individual caregivers, in terms of their emotional well-being (Hoening Hamilton 1966, Marx et al. 1973, Fadden et al. 1987, Gopinath \& Chaturverdi 1992, Winefield \& Harvey 1993, Schene et al. 1998, Friedrich et al. 1999, Provencher et al. 2003). However, although some studies have shown that the patient's participation in psychosocial rehabilitative activities or community based aftercare programmes, have a positive effect on their family environment, due to the patient's clinical stability and improvement of functioning, fewer investigators have explored this relationship within the framework of an experimental design (El-Islam 1982, Madianos \& Madianou 1992, Tomaras et al. 1988, Solomon et al. 1988, Halford \& Hays 1991, Corwall \& Scott 1996).

This study aims to explore the possible influence of rehabilitation of chronic schizophrenic patients on their family atmosphere and the emotional well-being of their key-relative-caregiver, compared to patients with the same sociodemographic and clinical characteristics, who were maintained on a regular ambulatory basis. Within the framework of psychosocial rehabilitation several clinical parameters were also examined namely: severity of diagnosis, medication compliance, overall psychosocial functioning of the participating patient.

\section{Methods}

\section{Participants}

Sixty six patients suffering from schizophrenia who were participating in psychosocial rehabilitation activities, in two day hospitals and three prevocational training workshops, in two psychiatric conglomerates in greater Athens respectively (group A) were randomly selected. From every rehabilitation programme an almost equal number of patients were selected. Seventy patients with the same diagnosis and sociodemographic characteristics, not attending any rehabilitation programme, were also randomly selected from the same psychiatric conglomerates (group B). These patients were under regular ambulatory maintenance treatment. For each patient of group A and B, a caregiver was selected for a personal face to face interview, after written consent was obtained. The study was approved by the ethical committee of each psychiatric center. Response rate was $100 \%$. 
The inclusion criteria for patients were the following: A diagnosis of schizophrenia according to DSM III-R criteria, duration of illness of at least 3 years, absence of any organic mental disorder or drug or alcohol abuse. Group A patients had a minimum participation of at least 6 months in rehabilitation and they were not hospital- ized during the 6 months prior to their admission to the programme. Group B patients had never participated in any rehabilitation programme.

The sociodemographic and clinical characteristics of both groups are presented in Table I.

Table I

Sociodemographic and clinical characteristics of group A (patients in rehabilitation) and group B (patients not in rehabilitation) $(\mathrm{n}=136)$

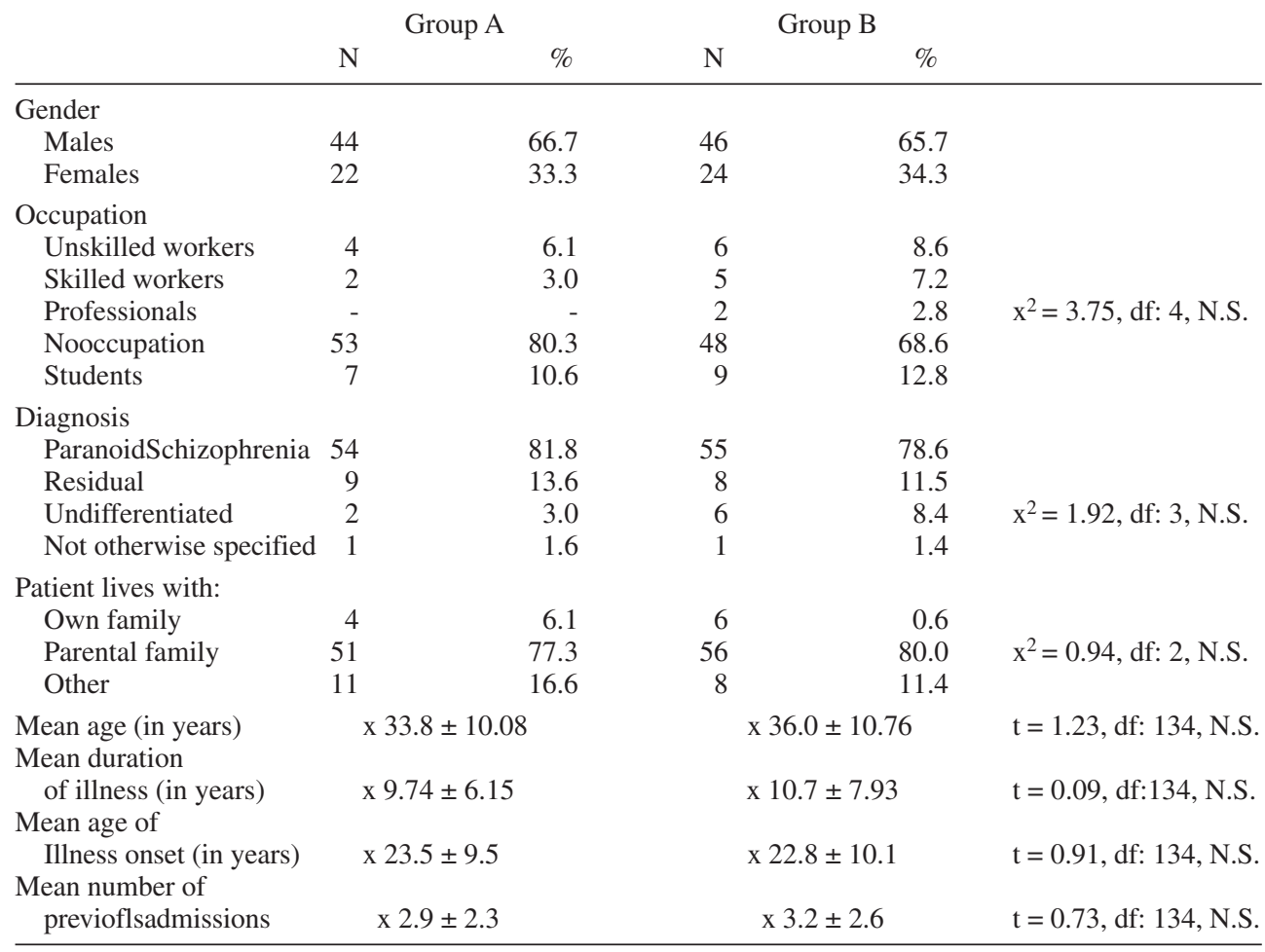

The mean age of group A and B patients was found to be $33.8( \pm 10.0)$ and 36.0 $( \pm 10.7)$ respectively and there is no statistical significant difference.

The majority of patients of both groups had no occupation and very few had had a job previous to their illness onset. The vast majority in both groups were single. With respect to the subtypes of diagnosis, the vast majority in both groups were diagnosed as suffering from "paranoid schizophrenia" with a second larger diagnostic proportion 
being that of "residual schizophrenia". Most of the patients of both groups were living with parental families.

The mean duration of illness in years was found to be $7.9( \pm 6.1)$ and $10.7( \pm 7.9)$ for group A and B of patients respectively, this was not statistically significant. The average age of illness onset was $23.5( \pm 9.5)$ for group A and $22.8( \pm 10.1)$ for group B also not a statistically significant difference.

Finally, the mean number of previous hospitalizations was found to be $2.9( \pm 2.3)$ and $3.2( \pm 2.6)$ for group A and B respectively.

Inclusion criteria for caregivers: For each patient a caregiver was selected on the basis of the following criteria. Age over 18 years, in contact with the patient for at least 6 hours per day, for a minimum of five days a week, during the last 12 months. The caregiver was also in regular contact with the psychiatric service, taking care of medication related issues and looking after the patient's needs. The absence of any reported history of mental disorder, physical disease and drug and alcohol abuse was required. This information was cross checked from the family history record of each patient. No previous involvement in the care of any other family member suffering from disabling physical or mental illness and previous or current participation in a family support programme (selfhelp group, psychoeducation) was also a prerequisite.

Table II presents the sociodemographic characteristics of caregivers.

Table II

Sociodemographic characteristics of caregivers of group A and B $(n=136)$

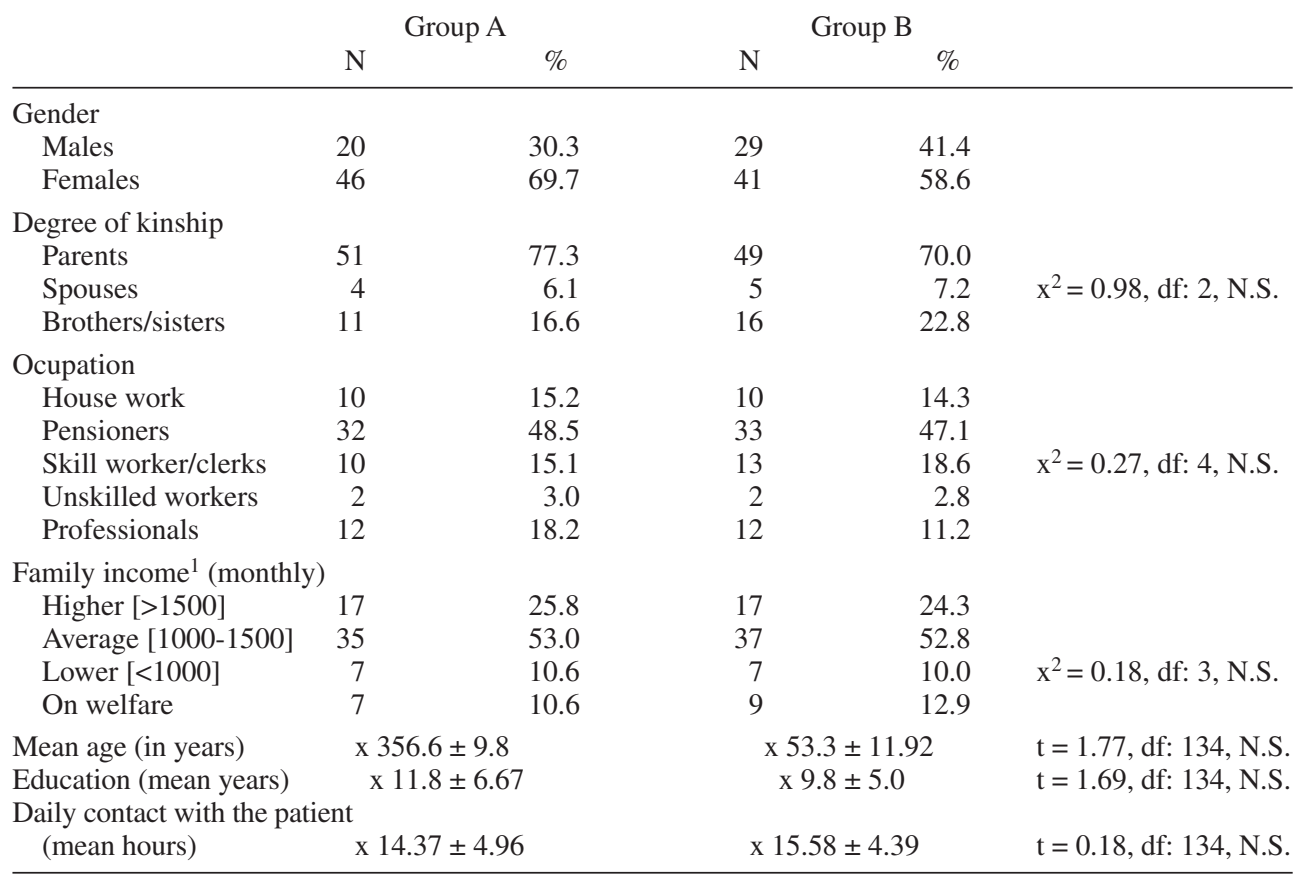

${ }^{1}$ In euros. 
A total of 136 family relatives -caregiverswere included in the study with a mean age of $56.6( \pm 9.8)$ years for group A and 53.3 $( \pm 11.9)$ years for group B caregivers, not a statistically significant difference.

The vast majority of caregivers for both groups were parents. An almost equal number of family members was found to be in both groups.

The average years of education for group A was $11.8( \pm 6.7)$ and $9.8( \pm 5.0)$ for group B. The majority of both groups of caregivers were pensioners or housewives. About half of the caregivers reported an income between 1000 and 1500 euros per month. On1y $25.8 \%$ of group A and $23.8 \%$ of group B families had a monthly income greater than 1500 euros.

Finally, the mean hours spent with the patient were found to be $14.3( \pm 4.9)$ for group A and $15.5( \pm 4.3)$ for group B, also not a statistically significant difference.

\section{Instruments}

The interview consisted of a sociodemographic data section for both patient and caregiver, as well as of additional psychometric scales.

These were the Global Assessment Scale (GAS) for evaluation of the patient's psychosocial functioning status (Endicott et al. 1976), and the Center for Epidemiological Studies - Depression scale (CES-D) for evaluation of the Primary caregiver's emotional well being.

The CES-D scale (Radloff 1977, Madianos \& Stefanis 1992) consists of 20 items representing major symptoms in the clinical syndrome of depression. In our study we modified the scale for personal interview and we employed a 4-week long criterion for symptom duration. The range of possible scores was from 0 - 60. This scale has been standardized with clinical and general population samples (Madianos \& Stefanis 1992).

A Global Assessment Schedule has been standardized with patients suffering from psychotic disorders (Madianos 1987). GAS ratings were made by the personal psychiatrist of each patient based on the patient's condition at the time of the study.

The overall family psychosocial environment was assessed by the interviewer by application of the Family Atmosphere Scale (FAS). This scale records quantitative scaled judgments of the patient's overall family environment. It measures six dimensions, namely the family's acceptance of the patient, economic problems, patient's dependency, medication compliance, intrafamilial relationships and family structure.

Each dimension is rated by a rater who knows the family dynamics, on a 4-point scale, so the total score ranges from 6 (positive family atmosphere) to 24 (negative family atmosphere). Standardization of the FAS in terms of internal consistency, concurrent and predictive validity and factor analysis was the subject of previous studies (Madianos et al. 1987; Madianos \& Madianou, 1992; Tomaras et al. 1988). All subjects of these studies were suffering from a schizophrenia spectrum of disorders, maintained on ambulatory basis. Their individual and family characteristics are compatible with those of the present study. Remainder correlations for each item had shown a statistically significant coefficient of alpha and negative family atmosphere was associated with higher relapse rate and readmission (Tomaras et al. 1988). Cut off scores $>12$ produced sensitivity $(81.0 \%)$ and specificity rates $(78.3 \%)$. 
The diagnosis of schizophrenia was established by the Structured Clinical Interview for DSM III R (SCID-I). The SCID I is a semistructured interview used for making an axis I DSM III R diagnosis (Spitzer et al. 1992). It has been translated and standardized for the Greek population (Madianos et al. 1997).

\section{Statistical analysis}

The possible statistical differences between group A and B of patients and caregivers mean values of non categorical variables were tested by the application of Student $\mathrm{t}$ - test. $\mathrm{X}^{2}$ statistic was also used to test the statistical significance of differences between group A and B of patients and caregivers categorical variables.

Finally, in the total sample $(\mathrm{n}=136)$ a stepwise multiple regression analysis was applied for detection of the possible effects of all independent variables on the Family
Atmosphere Scale and CES-D scale separately. The following independent variables were selected: patient's gender, diagnosis subtypes, medication compliance, participation of patient in rehabilitation, hours spent with the patient, caregiver' $s$ gender and education. The CES-D scale score (of caregivers) was included in the MRA with FAS as dependent variable. The FAS score and patient's global functioning (GAS score) were also included in the MRA with CES-D scale score of caregivers as dependent variable.

The statistical analysis was made by the use of SPSS 8.0 for Windows (1998).

\section{Results}

Table III presents the mean values of the Family Atmosphere Scale in groups A and B. The difference between these values was

Table III

Average score of Family Atmosphere Scale in groups A and B patients

\begin{tabular}{lcccccc} 
Groups of carevigers & $\mathrm{N}$ & $\mathrm{X}$ & $\mathrm{SD}$ & $\mathrm{t}$ value & $\mathrm{df}$ & $\mathrm{P}(2$ tailed $)$ \\
\hline $\mathrm{A}$ & 66 & 11.30 & 3.03 & 5.29 & 134 & $<.0001$ \\
$\mathrm{~B}$ & 70 & 14.31 & 3.70 & & &
\end{tabular}

found to be statistically significant at $\mathrm{p}<$ 0.001 implying that the families of psychotic patients in rehabilitation, were experiencing a lower level of family disruption and fewer psychosocial problems than their counterparts of group A families.

The application of stepwise multiple regression analysis revealed five indepen- dent variables, namely patient's gender, medication non compliance, hours spent with the patient, participation of the patient in a rehabilitation programme and the emotional status of caregivers (CES-D scale score) to predict the formation of the overall family atmosphere with total variance explained $41.5 \%$ (Table IV). These results could be interpreted as follows: 
Table IV

Stepwise multiple regression analysis results: Dependent variable: Family Atmospher Scale $(n=136)$

\begin{tabular}{lccccc} 
Independent variables & $\mathrm{R}^{2}$ & $\mathrm{BETA}$ & $\mathrm{SE}$ & $\mathrm{t}$ value & $\mathrm{P}$ value \\
\hline Patients gender & .03 & -.18 & .65 & -2.27 & $<.02$ \\
Medication compliance & .25 & .50 & .21 & 7.01 & $<.0001$ \\
Participation in a rehabilition programme & .08 & -.28 & .24 & -3.61 & $<.0001$ \\
Hours with the patient & .04 & .22 & .07 & 2.73 & $<.0001$ \\
Careviger's CES-D score & .38 & .62 & .02 & 9.49 & $<.0001$ \\
\hline
\end{tabular}

Total variance $\left(\mathrm{R}^{2}\right)=41.5 \%$

Female patients, non compliant with medication not in participation in a rehabilitation programme with a caregiver who spent more hours with them who is not emotionally well (high CES-D scale score), live in families exhibiting more disruption and psychosocial problems.

The average scores of CES-D scale (emotional well-being) in groups $\mathrm{A}$ and $\mathrm{B}$ caregivers are presented in Table V.

Table V

Average score of CES-D scale in groups A and B of caregivers

\begin{tabular}{lcccccc} 
Groups of carevigers & $\mathrm{N}$ & $\mathrm{X}$ & $\mathrm{SD}$ & $\mathrm{t}$ value & $\mathrm{df}$ & $\mathrm{P}(2$ tailed $)$ \\
\hline $\mathrm{A}$ & 60 & 17.34 & 7.74 & 11.65 & 144 & $<.0001$ \\
$\mathrm{~B}$ & 50 & 33.65 & 8.92 & & &
\end{tabular}

Group A caregivers with a relative-patient participating in a rehabilitation programme were found to exhibit fewer depressive symptoms than their counterparts of group B, at a statistically significant level $(\mathrm{p}<0.0001)$.

Finally, the stepwise regression analysis results of CES-D scale score of caregivers $(\mathrm{n}=136)$ are shown in Table VI.
The patients gender (females), the diagnostic subtype other than paranoid, medication non compliance, non participation in a rehabilitation programme, more hours spent with the patient, the caregiver's gender (females) and their lower level of education, a lower global psychosocial functioning of relativepatient (low GAS score) and a negative fami-

Table VI

Multiple regression analysis results: Dependent varibale : CES-D score of caregivers $(n=136)$

\begin{tabular}{llcccc} 
Independent variables & $\mathrm{R}^{2}$ & BETA & SE & t value & P value \\
\hline Patients gender & .02 & -.16 & 2.06 & -2.01 & $<.04$ \\
Diagnostiv subtypes & .02 & .15 & .93 & 1.93 & $<.05$ \\
Medication compliance & .39 & .62 & .60 & 9.60 & $<.0001$ \\
Participation in a rehabilition programme & .30 & -.55 & .68 & -7.94 & $<.0001$ \\
Hours spent with the patient & .26 & .51 & .21 & 7.28 & $<.0001$ \\
Careviger's gender & .05 & .22 & 2.00 & 2.78 & $<.0001$ \\
Careviger's education (years) & .05 & -.23 & .70 & -2.94 & $<.0001$ \\
FAS score & .38 & .62 & .20 & 9.49 & $<.0001$ \\
GAS score (patient) & .46 & -.67 & .03 & -11.07 & $<.001$ \\
\hline
\end{tabular}

Total variance $\left(\mathrm{R}^{2}\right)=76.0 \%$ 
ly atmosphere (high FAS score) were found to predict the formation of depressive caseness of caregivers (high CES-D scale score) explaining $76 \%$ of the total variance.

\section{Discussion}

The possible effects of a rehabilitation process on individual patients suffering from chronic and severe mental illness and on the caregiver-relative as well, were examined in this study. Particularly the patient's participation in day care or prevocational training activities, was found to improve their overall familial atmosphere and the patient's global psychosocial functioning.

Several disruptive characteristics such as hostility toward the patient or negative feelings about the patient, being an economic burden, phenomena of dependency, non compliance, often encouraged by family members, together with a disorganization of roles assessed by the Family Atmosphere Scale were all present to a lower extent in the families with a relative-patient participating in rehabilitation, when compared with caregivers whose patients were only in regular aftercare treatment.

The beneficial effect of a rehabilitation process was also noticed in the patient's global psychosocial functioning and increased medication compliance. Our findings are compatible with those reported by other investigators (Bury et al. 1998, Tomaras et al. 1988). Contrary to those results Mc Carthy et al. (1989) found that Participation in rehabilitation increased social and financial problems in the family.

This finding is probably attributed to the selection of their sample consisting of patients with different clinical characteristics.
When multivariate statistics were applied in the total sample of caregivers Family Atmosphere Scale score as a dependent variable, for the delineation of possible predictors, it was not surprising to find that the overall family atmosphere was worsened by the frequency of hours spent with the patient. Previous studies concerning familial factors and schizophrenia have shown a positive correlation between the caregiver' s perceived burden and the number of hours spent with the patient (Winefield \& Harvey 1994, Magliano et al. 1998). A positive correlation of high CES-D scale score (caregiver's depressive caseness) with negative family atmosphere (high FAS score) was also found.

The decrease of depressive symptomatology recorded in group A caregivers was associated with a positive family atmosphere. It is well established that relatives of schizophrenic patients experience a variety of negative feelings, concerning the patient's condition and their caring role, suffering from psychological distress, depressive and anxiety symptoms (Provencher et al. 2003, Wuezker et al. 2002, Barrowclough et al. 2003, Schene et al. 1998, Song et al. 1997). In our study female patients suffering from a stigmatizing illness, were causing more pain and despair in their families, associated with family disruptive phenomena, and an increased depressive symptomatology in their caregivers.

With respect to other underlying factors predicting the formation of an emotional status (CES-D scale score) of the total sample of caregivers, patient's gender (females), suffering from a diagnostic subtype of certain severity, non compliant with medication and with more hours spent with the patients, as well as the caregiver's gender (females), of lower education along with high scores in FAS (negative familial atmosphere) and low score in GAS (low psychosocial functioning 
level of the patient) predicted $76 \%$ of the total variance of CES-D scale score or the emotional status of caregivers.

Psychosocial functioning, incorporating psychotic psychopathology, gender of the patient related to the family's expectations (females are more socially protected in Greece than males) (Madianos \& Madianou 1991) and non medication compliance were also associated with familial disruption and causation of psychological distress in previous studies (Wuerker et al. 2002, Schene et al. 1998, Song et al. 1997, Madianos \& Economou, 1988).

Our results are not conclusive. Further developments of this study may examine the possible long lasting effects of rehabilitation on families or rural-semi-rural families with different support systems and families with patients with a dual diagnosis may be included in future research.

The results of this study provide evidence of the beneficial effect of community based psychosocial rehabilitation in the microenvironment of chronic psychotic patients through specific sociotherapeutic processes. However, our findings do not suggest that it is only the participation of a severely mentally ill person in rehabilitation that is needed for the improvement of family atmosphere but in those cases where specialized interventions are lacking, the involvement of family members in rehabilitation activities might also have a beneficial impact on their families.

\section{Acknowledgements}

This study is part of a research project funded by the Research Special Fund of University of Athens.

\section{References}

Barrowclough C, Tarrier N, Humphreys L, Ward J, Gregg L., Andrews B. Self-esteem in schizophrenia: relationships between self-evaluation, family attitudes, and symptomatology. J Abnorm Psychol 2003; 112: 92-99.

Brown GW, Birley JLT, Wing JK. Influence of family life on the course of schizophrenic disorders: a replication. Br J Psychiatry 1972; 121: 241-258.

Bury L, Zaborowski B, Konieczynska Z. Family burden in schizophrenic patients in different forms of psychiatric care. Psychiatr Pol 1998; 32: 275-286.

Corwall PL, Scott J. Burden of care, psychological distress and satisfaction with services in the relatives of acutely mentally disordered adults. Soc Psychiatry Psychiatr Epidemiol 1996; 31: 345-348.

Dyck DG, Short R, Vialiano PP. Predictors of burden and infectious illness in schizophrenia caregivers. Psychosom Med 1999; 61(4): 411-419.

El-Islam F. Rehabilitation of schizophrenics by the extended family. Acta Psychiatr Scand 1982; 65: 112-119.

Endicott J, Spitzer RL, Fleiss JL, Cohen J. The global assessment scale. A procedure for measuring overall severity of psychiatric disturbance. Arch Gen Psychiatry 1976; 33: 766-771.

Fadden G, Bebbington P, Kuipers L. The burden of care: the impact of functional psychiatric illness on the patient's family. Br J Psychiatry 1987; 150: 285-292.

Friedrich RM, Lively S, Buckwalter KC. well siblings living with schizophrenia. Impact of associated behaviors. J Psychosoc Nurs Ment Health Serv 1999; 37: 11-19.

Gopinath PS, Chaturverdi SK. Distressing behaviour of schizophrenics at home. Acta Psychiatr Scand 1992; 86: 185-188.

Halford W, Hayes R. Psychosocial rehabilitation of chronic schizophrenic Patients: recent findings on social skills training and family psychoeducation. Clinical Psychol Rev 1991; 11: 23-44.

Hoening J, Hamilton MW. The schizophrenic patient in the community and his effect on the household. Int $J$ Soc Psychiatry 1966; 12: 165-176.

Hogarty GE, Anderson CM, Reiss DJ et al. Family psychoeducation, social skills training and maintenance chemotherapy in the aftercare treatment of schizophrenia. I one-year effects of a controlled study on relapse and expressed emotion. Arch Gen Psychiatry 1986; 43: 633642 . 
Laidlaw TM, Coverdale JH, Falloon IR et al. Caregivers' stresses when living together or apart from patients with chronic schizophrenia. Community Ment Health J 2002; 38: 303-310.

Maccarthy B, Lesage A, Brewin CR. Needs for care among the relatives of long-term users of day care. A report from the camberwell high contact survey. Psychol Med 1989; 19: 725- 736.

Madianos M, Economou M. Negative symptoms in schizophrenia: the effect of long-term, community-based psychiatric intervention. Int J Ment Health 1988; 17: 2234.

Madianos M, Gournas G, Tomaras V, Kapsali A, Stefanis CN. Family atmosphere on the course of chronic schizophrenia treated in a community mental health center: a prospective longitudinal study. In. Stefanis Cn, editor. Schizophrenia: recent Biosocial developments. New York: Human Sciences Press; 1988. p. 246-256.

Madianos M, Madianou D. The greek family paradigm: implications for family therapy. Neurol Psychiatr 1991; 12: 132-138.

Madianos M, Madianou D. The effects of long-term community care on relapse and adjustment of persons with chronic schizophrenia. Int J Ment Health 1992; 21: 37-49.

Madianos M, Papaghelis M, Filippakis A. et al. The reliability of SCID I in Greece in clinical and general population. Psychiatriki 1997; 8: 101-108 (in Greek).

Madianos M, Stefanis C. Changes in the prevalence of symptoms of depression and depression across greece. Soc Psychiatry Psychiatr Epidemiol 1992; 27: 211-219.

Magliano L, Fadden G, Madianos M et al. Burden on the families of patients with schizophrenia: results of the biomed i study. Soc Psychiatry Psychiatr Epidemiol 1998; 33: 405-412.

Marx AJ, Test MA, Stein LI. Extra-hospital management of severe mental illness: feasibility and affects of social functioning. Arch Gen Psychiatry 1973; 29: 505511.

Platt S. Measuring the burden of psychiatric illness on the family: an evaluation of soma rating scales. Psychol Med 1985; 15: 383-393.

Provencher HL, Perreault M, St-Onge M, Rousseau M. Predictors of psychological distress in family caregivers of persons with psychiatric disabilities. J Psychiatr Ment Health Nurs 2003; 10: 592-607.
Radloff LS. The CES-D scale: a self-report depression scale for research in the general population. Appl Psycol Meas 1997; 1: 385-407.

Schene AH. Objective and subjective dimensions of family burden. Soc Psychiatry Psychiatr Epidemiol 1990; 25: 289-297.

Schene AH, Van Wijngaarden B, Koeter MWJ. Family caregiving in schizophrenia: domains and distress. Schizophr Bull 1998; 24: 609-618.

Solomon P, Draine J. Subjective burden among family members of mentally ill adults: relation to stress, coping and adaptation. Am J Orthopsychiatry 1995; 65: 419-427.

Song LY, Biegel DE, Milligan SE. Predictors of depressive symptomatology among lower social class caregivers of persons with chronic mental illness. Community Ment Health J 1997; 33: 269-286.

Spitzer RL, Williams JB, Gibbon M, First MB. The structured clinical interview for DSM-III-R (scid): history, rationale, and description. Arch Gen Psychiatry 1992; 49: 624-629.

Spss 8.0 For Windows Chicago, Spss Inc., 1998.

Tomaras V, Vlachonikolis IG, Stefanis CN, Madianos $M$. The effect of individual psychosocial treatment on the family atmosphere of schizophrenic patients. Soc Psychiatry Psychiatr Epidemiol 1988; 23: 256-261.

Vaughn $\mathrm{C}$, Leff J. The influence of family and social factors on the course of psychiatric illness. Br J Psychiatry 1976; 129: 125-137.

Winefield HR, Harvey EJ. Determinants of psychological distress in relatives of people with chronic schizophrenia. Schizophr Bull 1993; 19: 619-625.

Wuerker AK, Fu VK, Haas GL, Bellack AS. Age, expressed emotion, and interpersonal control patterning in families of person with schizophrenia. Psychiatr Res 2002; 109: 161-170.

Address of correspondence:

Professor M.G. Madianos,

Zografou Community Mental

Health Center 42 Davaki-Pindou Str,

Zografou 157- 73 Athens,

GREECE

e-mail: madianos@nurs.uoa.gr 